\title{
CONFORMAL AND KILLING VECTOR FIELDS ON REAL SUBMANIFOLDS OF THE CANONICAL COMPLEX SPACE FORM $\mathbb{C}^{m}$
}

\author{
HANAN ALOHALI, HAILA ALODAN, AND SHARIEF DESHMUKH
}

\begin{abstract}
In this paper, we find a conformal vector field as well as a Killing vector field on a compact real submanifold of the canonical complex space form $\left(\mathbb{C}^{m}, J,\langle\rangle,\right)$. In particular, using immersion $\psi: M \rightarrow \mathbb{C}^{m}$ of a compact real submanifold $M$ and the complex structure $J$ of the canonical complex space form $\left(\mathbb{C}^{m}, J,\langle\rangle,\right)$, we find conditions under which the tangential component of $J \psi$ is a conformal vector field as well as conditions under which it is a Killing vector field. Finally, we obtain a characterization of $n$-spheres in the canonical complex space form $\left(\mathbb{C}^{m}, J,\langle\rangle,\right)$.
\end{abstract}

\section{INTRODUCTION}

Conformal vector fields and Killing vector fields play a vital role in geometry of a Riemannian manifold $(M, g)$ as well as in physics (cf. [13]). In geometry, these vector fields are used in characterizing spheres among compact or complete Riemannian manifolds (cf. 4-12]). A Killing vector field is said to be nontrivial if it is not parallel. The existence of a nontrivial Killing vector field on a compact Riemannian manifold constrains its geometry as well as its topology: it does not allow the Riemannian manifold $(M, g)$ to have nonpositive Ricci curvature and if $(M, g)$ is positively curved, its fundamental group has a cyclic subgroup (cf. [2]). In most of the cases, a conformal vector field or a Killing vector field on a Riemannian manifold $(M, g)$ is derived through treating it as a submanifold of a Euclidean space. For example, a unit sphere $S^{n}$ admits a conformal vector field that is tangential component of a constant vector field on the ambient Euclidean space $R^{n+1}$. Similarly, an odd dimensional unit sphere $S^{2 m-1}$ with unit normal vector field $N$ as a hypersurface of the canonical complex space form $\left(\mathbb{C}^{m}, J,\langle\rangle,\right)$ admits a Killing vector field $\xi=-J N$, where $J$ is the canonical complex structure on $\mathbb{C}^{m}$. Therefore it is an interesting question to find a conformal vector field as well as a Killing vector field on a real submanifold of a canonical complex space form

2010 Mathematics Subject Classification. 53C20, 53C40.

Key words and phrases. Canonical complex space form; Ricci curvature; Conformal vector fields; Killing vector fields; Real submanifolds.

This research project was supported by a grant from the "Research Center of the Female Scientific and Medical Colleges", Deanship of Scientific Research, King Saud University. 
$\left(\mathbb{C}^{m}, J,\langle\rangle,\right)$. A similar study is taken up in 1 for submanifolds in a Euclidean space. Given an $n$-dimensional real submanifold $(M, g)$ of the canonical complex space form $\left(\mathbb{C}^{m}, J,\langle\rangle,\right)$ with immersion $\psi: M \rightarrow \mathbb{C}^{m}$, we treat $\psi$ as the position vector field of points on $M$ in $\mathbb{C}^{m}$, and consequently we have the expression $J \psi=$ $v+\bar{N}$, where $v$ is the tangential component and $\bar{N}$ is the normal component of $J \psi$ on $M$. This gives a globally defined vector field $v$ on the real submanifold $M$.

In this paper, we study the above mentioned question for real submanifolds of the canonical complex space form $\left(\mathbb{C}^{m}, J,\langle\rangle,\right)$ and obtain conditions under which the vector field $v$ is a conformal vector field (Theorems $3.1,3.2$ or a Killing vector field (Theorems 4.1, 4.3). We also use this vector field $v$ to find a characterization of a sphere $S^{n}(c)$ of constant curvature $c$ in the canonical complex space form $\left(\mathbb{C}^{m}, J,\langle\rangle,\right)$ (cf. Theorem 5.1). It is worth noting that the existence of the Killing vector field $v$ not only restricts the geometry and topology of the real submanifold $M$ but also has an influence on the dimensions of both the real submanifold and the ambient canonical complex space form $\left(\mathbb{C}^{m}, J,\langle\rangle,\right)$ (cf. Corollary 4.2). Finally, at the end of this paper, we give an example of a real submanifold of $\left(\mathbb{C}^{m}, J,\langle\rangle,\right)$ on which $v$ is a nontrivial conformal vector field (that is, $v$ is not Killing) and another example of a real submanifold on which $v$ is nontrivial Killing vector field (that is, non-parallel).

\section{Preliminaries}

Let $M$ be an immersed $n$-dimensional real submanifold of the canonical complex space form $\left(\mathbb{C}^{m}, J,\langle\rangle,\right), J$ and $\langle$,$\rangle being the canonical complex structure and$ the Euclidean metric on $\mathbb{C}^{m}$ respectively. We denote by $\mathfrak{X}(M)$ the Lie algebra of smooth vector fields on $M$, by $\Gamma(v)$ the space of sections of the normal bundle $v$ of $M$, and by $\bar{\nabla}$ and $\nabla$ the Riemannian connections on $\mathbb{C}^{m}$ and on $M$ respectively. Then we have the following Gauss and Weingarten equations for the real submanifold $M$ (cf. [3]):

$$
\bar{\nabla}_{X} Y=\nabla_{X} Y+h(X, Y), \quad \bar{\nabla}_{X} N=-A_{N} X+\nabla_{X}^{\perp} N,
$$

$X, Y \in \mathfrak{X}(M), N \in \Gamma(v)$, where $h$ is the second fundamental form, $A_{N}$ is the Weingarten map with respect to the normal $N \in \Gamma(v)$, which is related to the second fundamental form $h$ by

$$
g\left(A_{N} X, Y\right)=\langle h(X, Y), N\rangle, \quad X, Y \in \mathfrak{X}(M),
$$

and $\nabla^{\perp}$ is the connection in the normal bundle $v$. The curvature tensor field $R$ of the real submanifold $M$ is given by

$$
R(X, Y) Z=A_{h(Y, Z)} X-A_{h(X, Z)} Y, \quad X, Y, Z \in \mathfrak{X}(M) .
$$

The Ricci tensor field of the real submanifold $M$ is given by

$$
\operatorname{Ric}(X, Y)=n g(h(X, Y), H)-\sum_{i=1}^{n} g\left(h\left(X, e_{i}\right), h\left(Y, e_{i}\right)\right),
$$


where $\left\{e_{1}, \ldots, e_{n}\right\}$ is a local orthonormal frame on $M$ and

$$
H=\frac{1}{n} \sum_{i=1}^{n} h\left(e_{i}, e_{i}\right)
$$

is the mean curvature vector field of the real submanifold $M$.

The Ricci operator $Q$ is a symmetric operator defined by

$$
\operatorname{Ric}(X, Y)=g(Q(X), Y), \quad X, Y \in \mathfrak{X}(M) .
$$

Let $\psi: M \rightarrow \mathbb{C}^{m}$ be the immersion of the real submanifold $M$. Then we set

$$
J \psi=v+\bar{N},
$$

where $v$ is the tangential component and $\bar{N}$ is the normal component of $J \psi$.

Now, define skew symmetric tensors $\varphi$ and $G$, and the tensors $\Psi$ and $F$ as follows:

$$
\begin{aligned}
& J X=\varphi X+F X, \quad X \in \mathfrak{X}(M), \\
& J N=\Psi N+G N, \quad N \in \Gamma(v),
\end{aligned}
$$

where

$$
\begin{array}{ll}
\varphi: \mathfrak{X}(M) \longrightarrow \mathfrak{X}(M), & F: \mathfrak{X}(M) \longrightarrow \Gamma(v), \\
\Psi: \Gamma(v) \longrightarrow \mathfrak{X}(M), & G: \Gamma(v) \longrightarrow \Gamma(v),
\end{array}
$$

that is, $\varphi X, \Psi N$ are the tangential components of $J X$ and $J N$ respectively and $F X, G N$ are the normal components of $J X$ and $J N$ respectively.

Define a symmetric tensor $C$ of type $(1,1)$ by $C(X)=A_{\bar{N}} X, X \in \mathfrak{X}(M)$, and a smooth function $E: M \rightarrow \mathbb{R}$ on the real submanifold $M$ by $E=\langle H, \bar{N}\rangle$. Then we have

$$
\operatorname{tr} C=n E .
$$

Lemma 2.1. Let $M$ be an $n$-dimensional real submanifold of the canonical complex space form $\left(\mathbb{C}^{m}, J,\langle\rangle,\right)$. Then

$$
\nabla_{X} v=\varphi X+C(X) \quad \text { and } \quad \nabla_{X}^{\perp} \bar{N}=F X-h(X, v) .
$$

Proof. As $J$ is a complex structure, we have

$$
\bar{\nabla}_{X} J \psi=J \bar{\nabla}_{X} \psi
$$

which in view of equation 2.1 gives

$$
\nabla_{X} v+h(X, v)+\nabla_{X}^{\perp} \bar{N}-C(X)=\varphi X+F X, \quad X \in \mathfrak{X}(M) .
$$

Equating the tangential and the normal components we get the result.

Lemma 2.2. Let $M$ be an $n$-dimensional real submanifold of the canonical complex space form $\left(\mathbb{C}^{m}, J,\langle\rangle,\right)$. Then for $X, Y \in \mathfrak{X}(M)$ and $N \in \Gamma(v)$, we have

$$
\begin{aligned}
(\nabla \varphi)(X, Y) & =A_{F(Y)} X+\Psi(h(X, Y)), \text { where }(\nabla \varphi)(X, Y)=\nabla_{X} \varphi Y-\varphi \nabla_{X} Y \\
\left(D_{X} F\right) Y & =G(h(X, Y))-h(X, \varphi Y), \text { where }\left(D_{X} F\right) Y=\nabla_{X}^{\perp} F Y-F\left(\nabla_{X} Y\right) \\
\left(D_{X} \Psi\right) N & =A_{G(N)} X-\varphi A_{N} X, \text { where }\left(D_{X} \Psi\right) N=\nabla_{X} \Psi(N)-\Psi\left(\nabla_{X}^{\perp} N\right) \\
\left(\nabla_{X}^{\perp} G\right) N & =F\left(A_{N} X\right)-h(X, \Psi(N)), \text { where }\left(\nabla_{X}^{\perp} G\right) N=\nabla_{X}^{\perp} G N-G\left(\nabla_{X}^{\perp} N\right) .
\end{aligned}
$$


Proof. As $J$ is parallel, we have

$$
\bar{\nabla}_{X}(\varphi Y+F(Y))=J\left(\nabla_{X} Y+h(X, Y)\right),
$$

which in view of equation (2.1) takes the form

$$
(\nabla \varphi)(X, Y)+\left(D_{X} F\right) Y=A_{F(Y)} X+\Psi(h(X, Y))+G(h(X, Y))-h(X, \varphi Y),
$$

which on equating the tangential and the normal components gives the first two relations. Similarly, on using $\left(\bar{\nabla}_{X} J\right) N=0$, we get the remaining two.

Using Lemma 2.1. we find the divergence of the vector field $v$ as $\operatorname{div} v=n E$ and consequently, we have the following:

Lemma 2.3. Let $M$ be an $n$-dimensional compact real submanifold of the canonical complex space form $\left(\mathbb{C}^{m}, J,\langle\rangle,\right)$. Then

$$
\int_{M} E d V=0 \text {. }
$$

The following lemma is an immediate consequence of Lemma 2.1 .

Lemma 2.4. Let $M$ be an n-dimensional real submanifold of the canonical complex space form $\left(\mathbb{C}^{m}, J,\langle\rangle,\right)$. Then the tensor $C$ satisfies

(i) $(\nabla C)(X, Y)-(\nabla C)(Y, X)=R(X, Y) v+(\nabla \varphi)(Y, X)-(\nabla \varphi)(X, Y)$,

(ii) $\sum_{i=1}^{n}(\nabla C)\left(e_{i}, e_{i}\right)=n \nabla E+Q(v)+\sum_{i=1}^{n}(\nabla \varphi)\left(e_{i}, e_{i}\right)$,

where $(\nabla C)(X, Y)=\nabla_{X} C(Y)-C\left(\nabla_{X} Y\right), \quad X, Y \in \mathfrak{X}(M)$, and $\left\{e_{1}, \ldots, e_{n}\right\}$ is a local orthonormal frame of $M$.

Lemma 2.5. Let $M$ be an $n$-dimensional real submanifold of the canonical complex space form $\left(\mathbb{C}^{m}, J,\langle\rangle,\right)$. Then the skew symmetric tensor $\varphi$ satisfies

(i) $(\nabla \varphi)(X, Y)-(\nabla \varphi)(Y, X)=A_{F Y} X-A_{F X} Y$,

(ii) $\sum_{i=1}^{n}(\nabla \varphi)\left(e_{i}, e_{i}\right)=n \Psi(H)+\sum_{i=1}^{n} A_{F e_{i}} e_{i}$, where $X, Y \in \mathfrak{X}(M)$ and $\left\{e_{1}, \ldots, e_{n}\right\}$ is a local orthonormal frame of $M$.

Proof. (i) Using Lemma 2.2, we get

$$
\begin{aligned}
(\nabla \varphi)(X, Y)-(\nabla \varphi)(Y, X) & =A_{F Y} X+\Psi(h(X, Y))-A_{F X} Y-\Psi(h(Y, X)) \\
& =A_{F Y} X-A_{F X} Y, \quad X, Y \in \mathfrak{X}(M) .
\end{aligned}
$$

(ii) As $\operatorname{tr} \varphi=0$, we have

$$
\sum_{i=1}^{n} g\left((\nabla \varphi)\left(X, e_{i}\right), e_{i}\right)=0
$$

which gives

$$
\sum_{i=1}^{n}\left\{g\left((\nabla \varphi)\left(e_{i}, X\right), e_{i}\right)+g\left(A_{F e_{i}} X, e_{i}\right)-g\left(A_{F X} e_{i}, e_{i}\right)\right\}=0
$$

that is,

$$
\sum_{i=1}^{n}\left\{g\left(-(\nabla \varphi)\left(e_{i}, e_{i}\right)+A_{F e_{i}} e_{i}, X\right)+g(n \Psi(H), X)\right\}=0
$$


Hence,

$$
\sum_{i=1}^{n}(\nabla \varphi)\left(e_{i}, e_{i}\right)=n \Psi(H)+\sum_{i=1}^{n} A_{F e_{i}} e_{i} .
$$

Lemma 2.6. Let $M$ be an $n$-dimensional compact real submanifold of the canonical complex space form $\left(\mathbb{C}^{m}, J,\langle\rangle,\right)$. Then

$$
\int_{M}\left(\operatorname{Ric}(v, v)+\|C\|^{2}-\|\varphi\|^{2}-n^{2} E^{2}\right) d V=0 .
$$

Proof. Using Lemmas 2.4 and 2.5, we get

$$
\begin{gathered}
\operatorname{div} \varphi v=-\sum_{i=1}^{n} g\left(A_{F\left(e_{i}\right)} e_{i}, v\right)-n g(\Psi(H), v)-\|\varphi\|^{2}, \\
\operatorname{div} C v=\operatorname{Ric}(v, v)+n v(E)+n g(\Psi(H), v)+\|C\|^{2}+\sum_{i=1}^{n} g\left(A_{F e_{i}} e_{i}, v\right),
\end{gathered}
$$

and

$$
\operatorname{div} E v=v(E)+n E^{2}
$$

Using these equations, we conclude that

$$
\operatorname{div} C v=\operatorname{Ric}(v, v)+n \operatorname{div} E v-n^{2} E^{2}-\operatorname{div} \varphi v-\|\varphi\|^{2}+\|C\|^{2},
$$

which on integration gives the result.

Lemma 2.7. Let $M$ be an $n$-dimensional compact real submanifold of the canonical complex space form $\left(\mathbb{C}^{m}, J,\langle\rangle,\right)$. If $v$ satisfies $\Delta v=-\lambda v$ for a constant $\lambda>0$, where $\triangle$ is the Laplace operator acting on smooth vector fields on $M$, then

$$
\int_{M}\left\{\operatorname{Ric}(v, v)+\lambda\|v\|^{2}-2\|\varphi\|^{2}-n^{2} E^{2}\right\} d V=0 .
$$

Proof. Using the definition of the operator $C$ and Lemma 2.1 we have

$$
\begin{aligned}
(\nabla C)(X, Y) & =\nabla_{X} C Y-C \nabla_{X} Y \\
& =\nabla_{X}\left(\nabla_{Y} v-\varphi Y\right)-\nabla_{\nabla_{X} Y} v+\varphi \nabla_{X} Y \\
& =\nabla_{X} \nabla_{Y} v-\nabla_{\nabla_{X} Y} v-(\nabla \varphi)(X, Y), \quad X, Y \in \mathfrak{X}(M) .
\end{aligned}
$$

Taking a local orthonormal frame $\left\{e_{1}, \ldots, e_{n}\right\}$, the above equation leads to

$$
\begin{aligned}
\sum_{i=1}^{n}(\nabla C)\left(e_{i}, e_{i}\right) & =\sum_{i=1}^{n}\left(\nabla_{e_{i}} \nabla_{e_{i}} v-\nabla_{\nabla_{e_{i}} e_{i}} v\right)-\sum_{i=1}^{n}(\nabla \varphi)\left(e_{i}, e_{i}\right) \\
& =\triangle v-\sum_{i=1}^{n}(\nabla \varphi)\left(e_{i}, e_{i}\right) \\
& =-\lambda v-\sum_{i=1}^{n}(\nabla \varphi)\left(e_{i}, e_{i}\right),
\end{aligned}
$$

where we used the definition of the Laplace operator acting on smooth vector fields. 
Now, using Lemma 2.4 (ii) and Lemma 2.5, we conclude

$$
-\lambda\|v\|^{2}=\operatorname{Ric}(v, v)+n v(E)+2 g\left(\sum_{i=1}^{n} A_{F\left(e_{i}\right)} e_{i}, v\right)+2 n g(\Psi(H), v),
$$

and this equation together with equations $\sqrt{2.2}$ and $(2.3)$ by integration gives

$$
\int_{M}\left\{\operatorname{Ric}(v, v)+\lambda\|v\|^{2}-2\|\varphi\|^{2}-n^{2} E^{2}\right\} d V=0 .
$$

\section{Submanifolds With $v$ AS A CONFORmal VECTOR Field}

Recall that a smooth vector field $\xi$ on a Riemannian manifold $(M, g)$ is said to be a conformal vector field if the flow of $\xi$ consists of conformal transformations of the Riemannian manifold $(M, g)$. Equivalently, a smooth vector field $\xi$ on a Riemannian manifold $(M, g)$ is a conformal vector field if there exists a smooth function $\rho$ on $M$ that satisfies $£_{\xi} g=2 \rho g$, where $£_{\xi} g$ is the Lie derivative of $g$ with respect to $\xi$. The smooth function $\rho$ is called the potential function of the conformal vector field $\xi$. A conformal vector field $\xi$ is said to be a non trivial conformal vector field if the potential function $\rho$ is not a constant. In this section, we find conditions under which the vector field $v$ on the real submanifold $M$ of the canonical complex space form $\left(\mathbb{C}^{m}, J,\langle\rangle,\right)$ is a conformal vector field.

Theorem 3.1. Let $M$ be an n-dimensional compact real submanifold of the canonical complex space form $\left(\mathbb{C}^{m}, J,\langle\rangle,\right)$. If the Ricci curvature $\operatorname{Ric}(v, v)$ of $M$ satisfies

$$
\operatorname{Ric}(v, v) \geq n(n-1) E^{2}+\|\varphi\|^{2},
$$

then $v$ is a conformal vector field on $M$.

Proof. Using Lemma 2.6, we have

$$
\int_{M}\left(\operatorname{Ric}(v, v)-n(n-1) E^{2}-\|\varphi\|^{2}+\|C\|^{2}-n E^{2}\right) d V=0,
$$

which together with the condition in the hypothesis and Schwarz's inequality $\|C\|^{2} \geq n E^{2}$ gives

$$
\operatorname{Ric}(v, v)=n(n-1) E^{2}+\|\varphi\|^{2} \quad \text { and } \quad\|C\|^{2}=n E^{2} .
$$

The second equality holds if and only if $C=E I$, and consequently, the first equation in Lemma 2.1 reads

$$
\nabla_{X} v=E X+\varphi X, \quad X \in \mathfrak{X}(M) .
$$

This equation proves that

$$
\left(£_{v} g\right)(X, Y)=2 E g(X, Y), \quad X, Y \in \mathfrak{X}(M),
$$

that is, $v$ is a conformal vector field with potential function $E$. 
Theorem 3.2. Let $M$ be an n-dimensional compact real submanifold of the canonical complex space form $\left(\mathbb{C}^{m}, J,\langle\rangle,\right)$. If the vector field $v$ is an eigenvector of the Laplace operator, $\Delta v=-\lambda v$, and the Ricci curvature $\operatorname{Ric}(v, v)$ satisfies

$$
\operatorname{Ric}(v, v) \geq n(n-2) E^{2}+\lambda\|v\|^{2},
$$

then $v$ is a conformal vector field.

Proof. Lemma 2.6 implies

$$
-\int_{M}\|\varphi\|^{2} d v=\int_{M}\left(-\operatorname{Ric}(v, v)-\|C\|^{2}+n^{2} E^{2}\right) d V,
$$

which in view of Lemma 2.7 gives

$$
\int_{M}\left(\operatorname{Ric}(v, v)-\lambda\|v\|^{2}+2\|C\|^{2}-n^{2} E^{2}\right) d V=0,
$$

that is,

$$
\int_{M}\left(\operatorname{Ric}(v, v)-\lambda\|v\|^{2}-n(n-2) E^{2}+2\left(\|C\|^{2}-n E^{2}\right)\right) d V=0 .
$$

Thus, using the hypothesis and Schwarz's inequality $\|C\|^{2} \geq n E^{2}$, we get

$$
\operatorname{Ric}(v, v)=n(n-2) E^{2}+\lambda\|v\|^{2} \quad \text { and } \quad\|C\|^{2}=n E^{2},
$$

that is, $C=E I$. Hence, by Lemma 2.1. we get that $v$ is a conformal vector field.

\section{Submanifolds With $v$ as a Killing Vector Field}

Recall that a smooth vector field $\xi$ on a Riemannian manifold $(M, g)$ is said to be a Killing vector field if the flow of $\xi$ consists of isometries of the Riemannian manifold $(M, g)$. Equivalently, a smooth vector field $\xi$ on a Riemannian manifold $(M, g)$ is a Killing vector field if $£_{\xi} g=0$. In this section, we find conditions under which the vector field $v$ on the real submanifold $M$ of the canonical complex space form $\left(\mathbb{C}^{m}, J,\langle\rangle,\right)$ is a Killing vector field.

Theorem 4.1. Let $M$ be an n-dimensional compact real submanifold of the canonical complex space form $\left(\mathbb{C}^{m}, J,\langle\rangle,\right)$. Suppose that $v$ satisfies

(i) $v$ is an eigenvector of the Laplace operator with eigenvalue $-\lambda$,

(ii) $\operatorname{Ric}(v, v) \geq n(n-1) E^{2}+\|\varphi\|^{2}$,

(iii) $\|\varphi\|^{2} \geq \lambda\|v\|^{2}$.

Then $v$ is a Killing vector field.

Proof. The condition (ii), in view of Theorem 3.1, implies that $v$ is a conformal vector field with $C=E I$ and

$$
\operatorname{Ric}(v, v)=n(n-1) E^{2}+\|\varphi\|^{2} .
$$


Now, the condition (i), $\Delta v=-\lambda v$, combined with Lemma 2.7 and the above conclusion, gives

$$
\int_{M}\left(n(n-1) E^{2}+\|\varphi\|^{2}+\lambda\|v\|^{2}-2\|\varphi\|^{2}-n^{2} E^{2}\right) d V=0,
$$

that is,

$$
\int_{M}\left(\left(\|\varphi\|^{2}-\lambda\|v\|^{2}\right)+n E^{2}\right) d V=0 .
$$

Using condition (iii), we conclude that $E=0$ and consequently $C=0$. Thus, Lemma 2.1 gives

$$
\nabla_{X} v=\varphi X, \quad X \in \mathfrak{X}(M),
$$

that is,

$$
\left(£_{v} g\right)(X, Y)=0, \quad X, Y \in \mathfrak{X}(M) .
$$

Hence, $v$ is a Killing vector field.

Corollary 4.2. Let $M$ be an n-dimensional compact real submanifold of the canonical complex space form $\left(\mathbb{C}^{m}, J,\langle\rangle,\right)$, with positive sectional curvature. Suppose that $v$ satisfies

(i) $v$ is an eigenvector of the Laplace operator with eigenvalue $-\lambda$, that is, $\Delta v=$ $-\lambda v$,

(ii) $\operatorname{Ric}(v, v) \geq n(n-1) E^{2}+\|\varphi\|^{2}$,

(iii) $\|\varphi\|^{2} \geq \lambda\|v\|^{2}$.

Then either $n$ is odd or $m \geq n$.

Proof. Notice that $n<2 m$. Suppose the conditions (i)-(iii) hold. Then equation (4.2) implies $E=0, \lambda\|v\|^{2}=\|\varphi\|^{2}$, and combining these with equation 4.1, we get

$$
\operatorname{Ric}(v, v)=\lambda\|v\|^{2}=\|\varphi\|^{2} .
$$

Now, consider the smooth function $f=\frac{1}{2}\|v\|^{2}$, which by Lemma 2.1 and $E=0$, gives the gradient $\nabla f=-\varphi v$, and we compute

$$
\Delta f=-\sum_{i=1}^{n} g\left(\nabla_{e_{i}} \varphi v, e_{i}\right)=-\sum_{i=1}^{n} g\left(\nabla_{e_{i}} \nabla_{v} v, e_{i}\right) .
$$

Note that $E=0$, as in the proof of Theorem 4.1, we get $C=0$ and thus, an easy computation on using Lemma 2.1 with $E=0$ gives

$$
R(X, v) v=\nabla_{X} \nabla_{v} v-\varphi^{2} X
$$

that is,

$$
R(X, v, v, X)=g\left(\nabla_{X} \nabla_{v} v, X\right)+\|\varphi X\|^{2} .
$$

This equation in view of equation 4.4 implies

$$
\operatorname{Ric}(v, v)=-\Delta f+\|\varphi\|^{2},
$$

which together with equation 4.3 gives $\Delta f=0$. Hence, $f$ is a constant, that is, $v$ has constant length and consequently, $\varphi v=0$. 
If $v=0$, then Lemma 2.1 implies $\varphi=0$, that is, $J \psi=\bar{N}$, which on taking covariant derivative and using Lemma 2.1 gives $J X=F X, X \in \mathfrak{X}(M)$, and we get that $M$ is a totally real real submanifold of $\mathbb{C}^{m}$. Hence, in this case we have $2 n \leq 2 m$.

If $v \neq 0$, as $v$ is a Killing vector field of constant length $v(p) \neq 0$ for each $p \in M$, and as $M$ is compact connected with positive sectional curvature, then $M$ is odd-dimensional (for on an even-dimensional compact connected manifold of positive sectional curvature a Killing vector field has a zero).

Theorem 4.3. Let $M$ be an $n$-dimensional compact real submanifold of the canonical complex space form $\left(\mathbb{C}^{m}, J,\langle\rangle,\right)$. Suppose that $v \neq 0$ is not closed and satisfies $\varphi v=0$, with Ricci curvature

$$
\operatorname{Ric}(v, v) \geq n(n-1) E^{2}+\|\varphi\|^{2} .
$$

Then $v$ is a Killing vector field of constant length.

Proof. As in Theorem 3.1. the condition $\operatorname{Ric}(v, v) \geq n(n-1) E^{2}+\|\varphi\|^{2}$ implies that $v$ is a conformal vector field and the following hold:

$$
\nabla_{X} v=\varphi X+E X, X \in \mathfrak{X}(M) \quad \text { and } \quad \operatorname{Ric}(v, v)=n(n-1) E^{2}+\|\varphi\|^{2} .
$$

Using the first equation in 4.5, we get

$$
R(X, Y) v=X(E) Y-Y(E) X+(\nabla \varphi)(X, Y)-(\nabla \varphi)(Y, X)
$$

which gives

$$
\operatorname{Ric}(Y, v)=-(n-1) Y(E)-g\left(Y, \sum_{i=1}^{n}(\nabla \varphi)\left(e_{i}, e_{i}\right)\right)
$$

that is,

$$
\operatorname{Ric}(v, v)=-(n-1) v(E)-g\left(v, \sum_{i=1}^{n}(\nabla \varphi)\left(e_{i}, e_{i}\right)\right) .
$$

Now, taking divergence on both sides of the equation $\varphi v=0$, in view of equation (4.5), we have

$$
-\|\varphi\|^{2}-g\left(v, \sum_{i=1}^{n}(\nabla \varphi)\left(e_{i}, e_{i}\right)\right)=0
$$

and inserting this equation in 4.6 leads to

$$
\operatorname{Ric}(v, v)=-(n-1) v(E)+\|\varphi\|^{2},
$$

which on comparing with the second equation in 4.5 implies

$$
v(E)=-n E^{2}
$$

Also, using $\varphi v=0$ in the first equation in 4.5 gives

$$
\nabla_{v} v=E v
$$

which in view of equations (4.5) and 4.8) leads to

$$
R(X, v) v=X(E) v+n E^{2} X-(\nabla \varphi)(v, X)-E \varphi X-\varphi^{2} X,
$$


which on taking the inner product with $v$ and using $\varphi\left(\nabla_{v} v\right)=0$ (outcome of equation (4.9)), gives $X(E)\|v\|^{2}+n E^{2} g(X, v)=0$, that is,

$$
\|v\|^{2} \nabla E=-n E^{2} v \text {. }
$$

Hence, as $v \neq 0$, we get $\varphi(\nabla E)=0$, and taking divergence on both sides of this equation leads to $\operatorname{div}(\varphi(\nabla E))=0$, that is,

$$
g\left(\nabla E, \sum_{i=1}^{n}(\nabla \varphi)\left(e_{i}, e_{i}\right)\right)=0
$$

which in view of equation 4.10 implies

$$
-n E^{2} g\left(v, \sum_{i=1}^{n}(\nabla \varphi)\left(e_{i}, e_{i}\right)\right)=0 .
$$

Using 4.7) in the above equation, we get

$$
n E^{2}\|\varphi\|^{2}=0
$$

and as $v$ is not closed, from above equation, we conclude that $E=0$, and thus equation (4.5) reads, $\nabla_{X} v=\varphi X, X \in \mathfrak{X}(M)$, which proves that $v$ is a Killing vector field.

Moreover, if $f=\frac{1}{2}\|v\|^{2}$, then we have

$$
X(f)=g(\varphi X, v)=0, \quad X \in \mathfrak{X}(M),
$$

that is, $v$ has constant length.

\section{A Characterization OF SPHeres}

In this section we consider an $n$-dimensional compact real submanifold $M$ of the canonical complex space form $\left(\mathbb{C}^{m}, J,\langle\rangle,\right)$, and prove the following characterization for the spheres.

Theorem 5.1. Let $M$ be an n-dimensional compact Einstein submanifold of the canonical complex space form $\left(\mathbb{C}^{m}, J,\langle\rangle,\right), n>2$. Suppose that $v$ satisfies

(i) $v$ is an eigenvector of the Laplace operator with eigenvalue $-\lambda<\frac{S}{n}$,

(ii) $\operatorname{Ric}(v, v) \geq n(n-1) E^{2}+\|\varphi\|^{2}$, where $S$ is the constant scalar curvature.

Then $M$ is isometric to the sphere $S^{n}(c)$, for a constant $c>0$.

Proof. Using Theorem 3.1. we get that $v$ is a conformal vector field on $M$ and equation 4.5 holds. Thus, using the first equation in (4.5), we conclude

$$
(\nabla \varphi)(X, Y)=\nabla_{X} \nabla_{Y} v-\nabla_{\nabla_{X} Y} v-X(E) Y, \quad X, Y \in \mathfrak{X}(M),
$$

where $(\nabla \varphi)(X, Y)=\nabla_{X} \varphi y-\varphi \nabla_{X} Y$. Taking sum in the above equation over a local orthonormal frame $\left\{e_{1}, \ldots, e_{n}\right\}$ on $M$ and using $\Delta v=-\lambda v$, we get

$$
\sum_{i=1}^{n}(\nabla \varphi)\left(e_{i}, e_{i}\right)=\Delta v-\nabla E=-\lambda v-\nabla E
$$


Also, using equation (5.1, we find

$$
(\nabla \varphi)(X, Y)-(\nabla \varphi)(Y, X)=R(X, Y) v+Y(E) X-X(E) Y
$$

which on choosing $X=e_{i}$ and taking the inner product with $e_{i}$ and adding these $n$ equations corresponding to a local orthonormal frame $\left\{e_{1}, \ldots, e_{n}\right\}$ on $M$, we get

$$
-g\left(\sum_{i=1}^{n}(\nabla \varphi)\left(e_{i}, e_{i}\right), Y\right)=\operatorname{Ric}(Y, v)+(n-1) Y(E),
$$

where we used the fact that $\varphi$ is skew-symmetric and consequently $\sum g\left(\varphi e_{i}, e_{i}\right)=0$, and that $g((\nabla \varphi)(X, Y), Z)=-g((\nabla \varphi)(X, Z), Y)$. Combining equations 5.2 and $(5.3)$, we arrive at

$$
Q(v)=\lambda v-(n-2) \nabla E .
$$

Moreover, $M$ being an Einstein manifold, $Q(v)=\frac{S}{n} v$, and thus using equation (5.4) we get

$$
\nabla E=-\frac{S-n \lambda}{n(n-2)} v
$$

and as $S$ is a constant, we have $\nabla E=-c v$ for a constant $c$. This leads to

$$
\nabla_{X}(\nabla E)=-c \nabla_{X} v=-c(E X+\varphi X),
$$

that is, the Hessian of the smooth function $E$ is given by

$$
\begin{gathered}
H_{E}(X, Y)=-c E g(X, Y)-c g(\varphi X, Y) a, \quad X, Y \in \mathfrak{X}(M), \\
H_{E}(X, Y)-H_{E}(Y, X)=2 c g(\varphi Y, X) .
\end{gathered}
$$

Since the Hessian is symmetric, we get $c g(\varphi Y, X)=0, X, Y \in \mathfrak{X}(M)$. However, condition (i) in the hypothesis does not allow $c=0$ (as $c=0$ implies $S=n \lambda$ ); consequently we get $\varphi=0$, which changes equation (5.5) to

$$
\nabla_{X}(\nabla E)=-c E X, \quad X \in \mathfrak{X}(M),
$$

where $c$ is a positive constant by condition (i). Hence, by Obata's Theorem (cf. [11]), we get that $M$ is isometric to $S^{n}(c)$.

\section{EXAMPLES}

In this section, we give two examples of real submanifolds of a canonical complex space form $\left(\mathbb{C}^{m}, J,\langle\rangle,\right)$, one admitting a conformal vector field that is not Killing and other admitting a Killing vector field that is not parallel.

(i) Consider

$$
S^{2 n}(c)=\left\{x=\left(x_{1}, \ldots, x_{2 n+1}\right) \in R^{2 n+1}:\|x\|=\frac{1}{\sqrt{c}}, c>1\right\}
$$

and an immersion $\psi: S^{2 n}(c) \rightarrow C^{n+1}$ defined by

$$
\psi(x)=\left(x_{1}, \ldots, x_{2 n+1}, \sqrt{1-\frac{1}{c}}\right),
$$


which is clearly a smooth immersion. Observe that

$$
T_{p}\left(S^{2 n}(c)\right)=\left\{X \in R^{2 n+1}:\langle X, p\rangle=0\right\} .
$$

The two orthogonal unit normals $N_{1}, N_{2}$ for the real submanifold $S^{2 n}(c)$ in $C^{n+1}$ are given by

and

$$
N_{1}=\left(-\sqrt{c-1} x_{1}, \ldots,-\sqrt{c-1} x_{2 n+1}, \frac{1}{\sqrt{c}}\right)
$$

$$
N_{2}=\left(x_{1}, \ldots, x_{2 n+1}, \sqrt{1-\frac{1}{c}}\right) .
$$

Also, the standard complex structure $J$ on $C^{n+1}$ gives

$$
J \psi=\left(-x_{n+2}, \ldots,-x_{2 n+1},-\sqrt{1-\frac{1}{c}}, x_{1}, \ldots, x_{n+1}\right)
$$

and it is easy to check that

$$
\left\langle J \psi, N_{1}\right\rangle=\sqrt{c} x_{n+1} \quad \text { and } \quad\left\langle J \psi, N_{2}\right\rangle=0 .
$$

Expressing $J \psi=v+\bar{N}$, where $v \in \mathfrak{X}\left(S^{2 n}(c)\right)$, we get

$$
v=J \psi-\sqrt{c} x_{n+1}\left(-\sqrt{c-1} x_{1}, \ldots,-\sqrt{c-1} x_{2 n+1}, \frac{1}{\sqrt{c}}\right),
$$

that is,

$$
\begin{aligned}
v= & \left(-x_{n+2}, \ldots,-x_{2 n+1},-\sqrt{1-\frac{1}{c}}, x_{1}, \ldots, x_{n+1}\right) \\
& +\left(\sqrt{c^{2}-c} x_{1} x_{n+1}, \ldots, \sqrt{c^{2}-c} x_{n+1} x_{2 n+1},-x_{n+1}\right) \\
= & \left(\sqrt{c^{2}-c} x_{1} x_{n+1}-x_{n+2}, \ldots, \sqrt{c^{2}-c} x_{n+1}^{2}-\sqrt{1-\frac{1}{c}},\right. \\
& \left.\sqrt{c^{2}-c} x_{n+1} x_{n+2}+x_{1}, \ldots, \sqrt{c^{2}-c} x_{n+1} x_{2 n+1}+x_{n}, 0\right) .
\end{aligned}
$$

Now, using expressions of $N_{1}$ and $N_{2}$ it is straightforward to show that

$$
A_{N_{1}}=\sqrt{c-1} I \text { and } A_{N_{2}}=-I \text {, }
$$

and consequently that

$$
A_{\bar{N}}=\sqrt{c^{2}-c} x_{n+1} I .
$$

This proves that the vector field $v$ given by equation 6.3 satisfies

$$
£_{v} g=2 \sqrt{c^{2}-c} x_{n+1} g,
$$

that is, $v$ is a conformal vector field. Note that this vector field is not a Killing vector field on $S^{2 n}(c)$. To verify the last assertion, we see from the last equation that if $v$ is Killing, $x_{n+1}=0$, and consequently equation 6.2 gives that $v=J \psi$. Moreover, $S^{2 n}(c)$ being an even-dimensional compact and connected manifold of 
positive sectional curvature, there would exist a point $p$ where $(J \psi)(p)=0$; using this in equation 6.1 we get $c=1$, a contradiction.

(ii) Consider the unit sphere $S^{2 n-1}$ in $R^{2 n}$ and an immersion $\psi: S^{2 n-1} \rightarrow \mathbb{C}^{m}$, $m>n$, defined by

$$
\psi\left(x_{1}, \ldots, x_{n}, \ldots, x_{2 n}\right)=\left(x_{1}, \ldots, x_{2 n}, c_{1}, \ldots, c_{2 m-2 n}\right),
$$

where $c_{i}, 1 \leq i \leq 2 m-2 n$, are constants and $\mathbb{C}^{m}$ is identified with $R^{2 m}$. A local frame of orthonormal normal vector fields for this immersion is given by $\left\{N_{1}, N_{2}, \ldots, N_{2 m-2 n+1}\right\}$, where

$$
N_{1}=\left(x_{1}, \ldots, x_{2 n}, 0, \ldots, 0\right)
$$

and

$$
N_{\alpha}=(0, \ldots, 0,1,0, \ldots, 0), 1 \text { at the }(2 n+\alpha)^{\text {th }} \text { place, } 2 \leq \alpha \leq 2 m-2 n+1 .
$$

Consider a complex structure $J$ on $\mathbb{C}^{m}$ defined by

$$
J E=\left(-E\left(x_{2}\right), E\left(x_{1}\right),-E\left(x_{4}\right), E\left(x_{3}\right), \ldots,-E\left(x_{2 m}\right), E\left(x_{2 m-1}\right)\right), \quad E \in \mathfrak{X}\left(\mathbb{C}^{m}\right),
$$

which makes $\left(\mathbb{C}^{m}, J,\langle\rangle,\right)$ a Kaehler manifold. Now set $J \psi=v+\bar{N}$, where $v \in$ $\mathfrak{X}\left(S^{2 n-1}\right)$ is the tangential component and $\bar{N}$ is the normal component of $J \psi$. We get

$$
\begin{array}{r}
J \psi=\left(-x_{2}, x_{1}, \ldots,-x_{2 n}, x_{2 n-1},-c_{2}, c_{1}, \ldots,-c_{2 m-2 n}, c_{2 m-2 n-1}\right), \\
\left\langle J \psi, N_{1}\right\rangle=0, \quad\left\langle J \psi, N_{\alpha}\right\rangle=-(-1)^{\alpha} c_{\alpha}, \quad 2 \leq \alpha \leq 2 m-2 n+1,
\end{array}
$$

and consequently,

$$
\bar{N}=\sum_{\alpha=1}^{2 m-2 n+1}\left\langle\bar{N}, N_{\alpha}\right\rangle N_{\alpha}=\left(0, \ldots, 0,-c_{2}, c_{1}, \ldots,-c_{2 m-2 n}, c_{2 m-2 n-1}\right) .
$$

Thus, equations (6.4) and 6.5 imply

$$
v=J \psi-\bar{N}=\left(-x_{2}, x_{1}, \ldots,-x_{2 n}, x_{2 n-1}, 0, \ldots, 0\right) .
$$

Let $\bar{\nabla}$ and $\nabla$ be the Euclidean connection on $\mathbb{C}^{m}$ and the Riemannian connection on the real submanifold $\left(S^{2 n-1}, g\right)$ with respect to the induced metric $g$. Then using equation 6.6 we get

$$
\begin{aligned}
\nabla_{X} v & =\bar{\nabla}_{X} v-h(X, v) \\
& =\left(-X\left(x_{2}\right), X\left(x_{1}\right), \ldots,-X\left(x_{2 n}\right), X\left(x_{2 n-1}\right), 0, \ldots, 0\right)-h(X, v),
\end{aligned}
$$

$X \in \mathfrak{X}\left(S^{2 n-1}\right)$, where $h$ is the second fundamental form. Taking the inner product with $Y \in \mathfrak{X}\left(S^{2 n-1}\right)$ in the above equation we arrive at

$$
g\left(\nabla_{X} v, Y\right)=-X\left(x_{2}\right) Y\left(x_{1}\right)+\cdots-X\left(x_{2 n}\right) Y\left(x_{2 n-1}\right)+X\left(x_{2 n-1}\right) Y\left(x_{2 n}\right),
$$

which leads to

$$
g\left(\nabla_{X} v, Y\right)+g\left(\nabla_{Y} v, X\right)=0, \quad X, Y \in \mathfrak{X}\left(S^{2 n-1}\right) .
$$

Thus, the vector field $v$ satisfies

$$
£_{v} g=0,
$$


that is, $v$ is a Killing vector field on $S^{2 n-1}$. That the Killing vector field $v$ is not parallel follows from equation 6.7, that is, $v$ is a nontrivial Killing vector field.

\section{REFERENCES}

[1] H. Alohali, H. Alodan and S. Deshmukh, Conformal vector fields on submanifolds of a Euclidean space, Publ. Math. Debrecen 91 (2017), no. 1-2, 217-233. MR 3690531

[2] V. Berestovskii and Y. Nikonorov, Killing vector fields of constant length on Riemannian manifolds, Siberian Math. J. 49 (2008), no. 3, 395-407. MR 2442533.

[3] B.Y. Chen, Total Mean Curvature and Submanifolds of Finite Type, World Scientific, Singapore, 1984. MR 0749575.

[4] S. Deshmukh, Characterizing spheres by conformal vector fields, Ann. Univ. Ferrara Sez. VII Sci. Mat. 56 (2010), no. 2, 231-236. MR 2733411

[5] S. Deshmukh, Conformal vector fields and eigenvectors of Laplacian operator, Math. Phys. Anal. Geom. 15 (2012), no. 2, 163-172. MR 2915600

[6] S. Deshmukh, A note on hypersurfaces in a sphere, Monatsh. Math. 174 (2014), no. 3, 413426. MR 3223496

[7] S. Deshmukh, Characterizations of Einstein manifolds and odd-dimensional spheres, J. Geom. Phys. 61 (2011), no. 11, 2058-2063. MR 2827109

[8] S. Deshmukh, F. Al-Solamy, Conformal gradient vector fields on a compact Riemannian manifold, Colloq. Math. 112 (2008), no. 1, 157-161. MR 2373435

[9] A. Lichnerowicz, Géométrie des groupes de transformations, Travaux et Recherches Mathématiques III, Dunod, Paris, 1958. MR 0124009

[10] M. Obata, Certain conditions for a Riemannian manifold to be isometric with a sphere, J. Math. Soc. Japan 14 (1962), 333-340 MR 0142086

[11] S. Tanno and W. Weber, Closed conformal vector fields, J. Diff. Geom. 3 (1969), 361-366. MR 0261498

[12] Y. Tashiro, On conformal and projective transformations in Kählerian manifolds, Tohoku Math. J. 14 (1962), 317-320. MR 0157339

[13] Y. Choquet-Bruhat, C. DeWitt-Morette, M. Dillard-Bleick, Analysis, Manifolds and Physics, North-Holland, New York-Oxford, 1977. MR 0467779

H. Alohali, H. Alodan, S. Deshmukh ${ }^{凶}$

Department of Mathematics, College of Science, King Saud University, P.O. Box-2455,

Riyadh-11451, Saudi Arabia

halohali@ksu.edu.sa

halodan1@ksu.edu.sa

shariefd@ksu.edu.sa

Received: November 6, 2018

Accepted: January 22, 2019 